\title{
Improving System Performance of Ad Hoc Wireless Network with Directional Antenna
}

\author{
S. Bandyopadhyay, M.N. Pal, Dola Saha \\ Indian Institute of Management Calcutta \\ Joka, Diamond Harbour Road \\ Calcutta 700104 INDIA
}

\author{
Tetsuro Ueda, Kazuo Hasuike \\ ATR Adaptive Communications \\ Research Laboratories \\ Seika-cho, Kyoto 619-0288, JAPAN
}

\author{
Ranjan Pal \\ Birla Institute of Technology \\ Meshra, Ranchi 835215 \\ INDIA
}

\begin{abstract}
It has been shown that use of directional antenna in the context of ad hoc wireless networks can largely reduce radio interference, thereby improving the utilization of wireless medium. However, that alone does not always guarantee improvement in overall system performance. In this paper, we have identified several criteria and investigated their interrelationships and impact on overall system performance in this context. Our methodology uses optimisation techniques using multicriteria decision analysis. We use analytic hierarchy process (AHP) to identify relative weights of different criteria under different application-specific scenario in order to solve the optimisation problem for each scenario by TOPSIS approach. The result shows that the parameter setting required to get optimum performance is application-specific; depending on the situation or application-scenario, several parameters need to be controlled to get better system performance.
\end{abstract}

\section{INTRODUCTION}

In ad hoc wireless networks [1], all the nodes are usually equipped with omni-directional antenna. However, ad hoc networks with omni-directional antenna uses omni-directional RTS/CTS based medium access mechanism that wastes a large portion of the network capacity by reserving the wireless media over a large area. As a result, lot of nodes in the neighborhood of transmitter and receiver has to sit idle, waiting for the data communication between transmitter node and receiver node to finish. To overcome this problem, researchers have proposed to use directional or adaptive antenna that would largely reduce radio interference, thereby improving the utilization of wireless medium and consequently the network throughput [2-5]. However, in the context of ad hoc networks, it is difficult to find ways to control the direction of such antenna for transmission and reception in each terminal in order to achieve an effective multi-hop communication between any source and destination. In other words, in order to fully exploit the capability of directional antenna, whenever a source $\mathrm{S}$ and destination $\mathrm{D}$ engage in a communication, all the neighbors of source and destination nodes should know the direction of communication so that they can initiate new communication in other directions. In [2], an adaptive MAC protocol has been proposed, where each node keeps certain neighborhood information dynamically through the maintenance of an Angle-SINR Table in order that each node knows the direction of communication events going on in its neighborhood at that instant of time.

However, periodic exchange of this information increases the control overhead of the system. If the system is highly mobile, then each node has to update this angle-specific neighborhood information more frequently, which would increase the control overhead further. Additionally, if the transmission beam-width of a transmitter node towards a receiver is narrow, the receiver may quickly move out of the transmission zone so formed and the communication would be interrupted.

Thus, even if we design effective MAC and routing protocols with directional antenna, that may not guarantee overall improvement in system performance. For example, what should be the optimum value of transmission-beam-angle $\alpha$ and transmission range $\mathrm{R}$ for better system performance? Will that be application-scenario-specific or it would be the same for every scenario? Assume that it is possible to get a directional antenna that can form a very narrow beam. Will that be more effective in every context?

In this paper, we have identified several criteria and investigated their inter-relationships and impact on overall system performance in this context. Our methodology uses optimisation techniques using multicriteria decision analysis. We use analytic hierarchy process (AHP) to identify relative weights of different criteria under different applicationspecific scenario in order to solve the optimisation problem for each scenario by TOPSIS approach. The results show that the parameter setting to get optimum performance is applicationspecific; depending on the situation or application-scenario, several criteria need to be controlled for better system performance.

\section{SYSTEM DESCRIPTION}

An ad hoc network consists of $\mathrm{N}$ nodes distributed over a twodimensional space of area A. Let us assume that each node $\mathrm{n}$ is equipped with a directional antenna whose beam-angle can vary from 30 degree to 360 degree. Each node has a transmission range $\mathrm{R}$ and is moving with a velocity $\mathrm{V}$ units/sec. within the specified area A.

Neighbors of $\mathbf{n}$ : Neighbors of $n \in N$ is a set of nodes within the omni-directional transmission range $\mathrm{R}$ of $\mathrm{n}$.

transmission_zone $(\boldsymbol{n})$ : When a node $\mathrm{n}$ forms a transmission beam with a beam-angle $\alpha\left(30^{\circ}<=\alpha<=360^{\circ}\right)$ and a 
transmission range $\mathrm{R}$ with respect to $\mathrm{n}$, the coverage area of $\mathrm{n}$ is defined as transmission_zone $\mathrm{n}_{\mathrm{n}}(\alpha)$. It implies that if a node $\mathrm{m} \in \mathrm{N}$ is within the transmission_zone $\mathrm{n}_{\mathrm{n}}(\alpha)$ and $\mathrm{m}$ is in omnidirectional receive mode, then, whenever $\mathrm{n}$ transmits a message at that transmission angle $\alpha$ with respect to $n$, it will be received by $m$. Else, the connectivity between $n$ and $m$ is lost. The area of transmission_zone $(\alpha)=\alpha \mathrm{R}^{2} / 2$.

Longevity (L): Longevity of node $m$ with respect to node $n$, $\mathrm{L}_{\mathrm{n}, \mathrm{m}}(\mathrm{t})$, is the life-span of the connectivity between $\mathrm{n}$ and $\mathrm{m}$. In other words, the amount of time node $\mathrm{m}$ remains within the transmission_zone $\mathrm{n}(\alpha)$ of node $\mathrm{n}$ is defined as longevity of node $m$ with respect to $n$. When the nodes are mobile, greater the angle $\alpha$, greater will be the longevity.

Medium Utilisation (MU): This is defined as the capacity of the system to support multiple number of simultaneous communications in the medium. As discussed in [2], we see that the smaller the transmission zone of a node, greater is the medium utilisation. Thus, for all $\mathrm{n} \in \mathrm{N}$, if transmission_zone $\mathrm{n}_{\mathrm{n}}$ $(\alpha)$ is small, medium utilisation will be high. So, medium utilisation has an inverse relationship with transmission_zone $\mathrm{n}_{\mathrm{n}}$ $(\alpha)$ and will be proportional to $1 /\left(\alpha . R^{2} / 2\right)$ i.e. $2 / \alpha . R^{2}$.

Cost of Antenna (CA): It has been assumed that the cost of antenna depends on the angular coverage and transmission range. In directional antenna, if we need to have low $\alpha$, it implies more number of antenna elements [6] and it would cost more. Again, greater the transmission range, greater is the cost of antenna, since more power would be needed to get a higher transmission range. Based on these observations, cost of antenna is defined as a linear function of $\alpha$ and $\mathrm{R}$.

Cost of Overhead (CO): Cost of overhead is defined as the overhead incurred in maintaining the angle-specific neighborhood information at each node. As indicated in [2], a node should know how to set its transmission direction to transmit a packet to its neighbors, avoiding interference with other nodes. So, each node periodically collects its neighborhood information and forms an Angle- SINR Table (AST) [2]. In a mobile environment, if $\alpha$ is small, then AST of a node needs to be changed very rapidly; therefore the overhead in maintaining the table also increases. So, greater the angle formed by the source node lesser is the overhead cost.

\section{MODEL DESCRIPTION}

We have identified four criteria and analysed their interrelationships and impact on the system performance. These criteria are: Longevity, medium utilisation, cost of antenna and cost of overhead. Ideally, we should have a system with high longevity, high medium utilisation, low antenna-cost and low overhead-cost. However, if we look at their interrelationships, we see that this cannot be achieved in a mobile environment of ad hoc networks. For example, if we need high medium utilisation, it implies that for all $n$, transmission_zone $e_{n}(\alpha)$ should be small, which in turn implies that the longevity will be low and antenna cost will be high. So, we cannot derive an optimum solution to get an optimum value of $\alpha$ and $R$ that would guarantee best system performance in every scenario.

Let the decision variables of the model are the transmission range $\mathrm{R}$ and antenna-beam-angle $\alpha$, forming a transmission_zone $\mathrm{n}_{\mathrm{n}}(\alpha)$ for any node $\mathrm{n}$. The objective functions of the model are Longevity (L), Medium Utilization (MU), Cost of Antenna (CA) and Cost of Overhead (CO) and are described as follows:

1. Longevity (L): To determine longevity of node $O$ w.r.t node $\mathrm{P}$, it is assumed that node $\mathrm{O}$ randomly chooses a direction and starts moving in that direction with a constant speed $\mathrm{v}$. The expected time taken by $\mathrm{O}$ to cross the boundary of the zone is longevity L (figure 1):

$$
\begin{aligned}
& L=t_{a v}=\frac{1}{\alpha R} \int_{0}^{\alpha} \int_{0}^{R} t(r, \theta) d r d \theta \text {, where }
\end{aligned}
$$

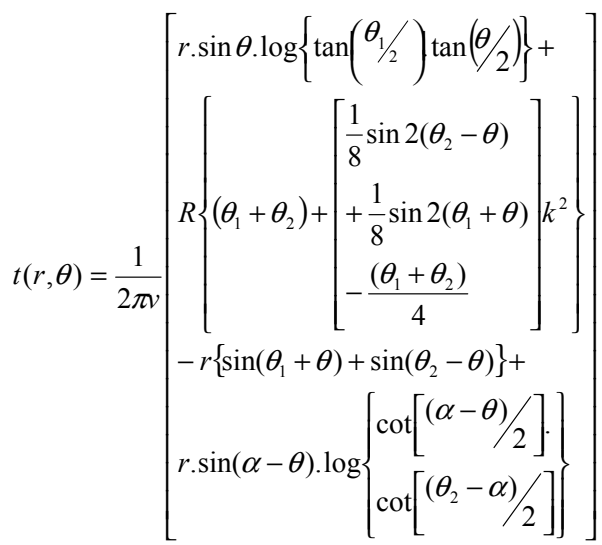

and $\cot \theta_{1}=\left(\frac{R}{r}-\cos \theta\right) \cdot \operatorname{cosec} \theta$

$$
\cot \theta_{2}=\frac{R \cos a-r \cos \theta}{R \sin a-r \sin \theta} \text { and } k=\frac{r}{R}
$$

The derivation of Longevity is not shown in this paper.

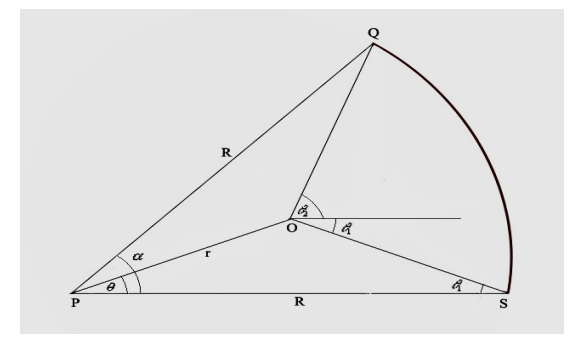

Figure 1. Determining Longevity of node $\mathrm{O}$ with respect to $\mathrm{P}$. 2. Medium Utilisation (MU): As defined earlier, MU is the inverse of the area of a transmission zone $(a)$.

$M U=K_{0} /\left(\frac{\alpha}{2 \pi} \cdot \pi R^{2}\right)=\frac{2 K_{0}}{R^{2} \alpha}$

3. Cost of Antenna (CA): As defined earlier,

$$
C A=K_{1}+K_{2} R+\frac{K_{3}}{a}
$$

4. Cost of Overhead (CO): As defined earlier,

$$
\mathrm{CO}=K_{4}+\frac{K_{5}}{a}
$$

The constraints are given as follows: 
$R_{\text {min }} \leq R \leq R_{\max }$

$\alpha_{\min } \leq \alpha \leq \alpha_{\max }$

$N_{\min } \leq N D \frac{R^{2} \alpha}{2} \leq N_{\max }$

where, ND $=$ Node Density of the application and

$K_{0}, K_{1}, K_{2}, K_{3}, K_{4}, K_{5}$ are constants.

So, the Multi-objective Optimization Model becomes

Maximize: $L=t_{a v}$, given $\mathrm{v}$

Maximize: $M U=\frac{2 K_{0}}{R^{2} a}$

Minimize: $C A=K_{1}+K_{2} R+\frac{K_{3}}{a}$

Minimize: $\mathrm{CO}=K_{4}+\frac{K_{5}}{a}$

Subject to

$R_{\min } \leq R \leq R_{\max }$

$\alpha_{\min } \leq \alpha \leq \alpha_{\max }$

$N_{\min } \leq N D \frac{R^{2} \alpha}{2} \leq N_{\max }$

where, $R_{\min }, R_{\max }=$ lower and upper bound of transmssion range

$\alpha_{\min }, \alpha_{\max }=$ lower and upper bound of $\alpha$

$N_{\min }, N_{\max }=$ lower and upper bound of number of nodes in a

transmission zone $(\alpha)$

\section{METHODOLOGY}

Our methodology uses optimisation techniques using multicriteria decision analysis. We use analytic hierarchy process (AHP) to identify relative weights of different criteria under different application-specific scenario in order to solve the optimisation problem for each scenario. Almost all the decision problems have multiple, usually conflicting criteria. These problems are usually solved either by Multiple Attribute Decision Making (MADM) method in which number of alternatives has been predetermined and the decision maker has to select/rank these alternatives or by Multiple Objective Decision Making (MODM) method in which the decision maker has to design a most promising alternative under resource and other constraints since predetermined alternatives are not available. Technique for Order Preference by Similarity to Ideal Solution (TOPSIS) and Analytic Hierarchy Process (AHP) are the two effective decision making tools for solving Multi Criteria Decision Making (MCDM) problems.

A. Technique for Order Preference by Similarity to Ideal Solution (TOPSIS)

The MCDM problem can then be solved by locating the alternative or decision relatively closest to the positive reference point and longest from the negative reference point. Hwang and Yoon [7,8] first developed this method for solving Multiple Attribute Decision Making (MADM) problems which is based upon the principle that the chosen alternative should have the shortest distance from the Positive Ideal Solution (PIS) and the longest distance from the Negative Ideal Solution (NIS). Quite often, the chosen alternative/decision is such that its distance is shortest from the PIS but not necessarily the longest from the NIS in comparison to the other alternatives/decisions. The principle of TOPSIS determines the solution where the distance of the chosen alternative/decision is relatively shortest from the PIS and longest from the NIS. The distance from the reference point is measured by using Minkwaski's Lp metric. The distance $d_{p}$ between two points $\boldsymbol{f}$ and $\boldsymbol{f}^{*}$ in metric in Kdimensional space is defined as:

$d_{p}=\left[\sum_{k=1}^{K}\left(f_{k}^{*}-f_{k}\right)^{p}\right]^{\frac{1}{p}}$, where $p \geq 1$ and $k=1,2, \ldots, K$

when $\mathrm{p}$ increases, $d_{p}$ decreases and a greater emphasis is

given to the largest deviation in forming the total.

In order to remove incommensurability among different dimensions, the normalization of $d_{p}$ is carried out as follows:

$d_{p}=\left\{\sum_{k=1}^{K}\left[\left(f_{k}^{*}-f_{k}\right) /\left(f_{k}^{*}-f_{k}^{-}\right)\right]^{p}\right\}^{\frac{1}{p}}$, for $p \geq 1$

where, $\quad f^{*}=$ Positive Ideal Solution (PIS), and

$\boldsymbol{f}^{-}=$Negative Ideal Solution (NIS)

The MODM problem can be defined as:

Maximize/Minimize $\left[f_{1}(\boldsymbol{x}), f_{2}(\boldsymbol{x}), \ldots, f_{k}(\boldsymbol{x})\right]=\boldsymbol{f}(\boldsymbol{x})$

subject to $\boldsymbol{x} \in X=\left\{\boldsymbol{x} \mid g_{s}(\boldsymbol{x})\{\geq,=, \leq\} 0, s=1,2, \ldots, m\right\}$.....

where, $f_{j}(\boldsymbol{x}), j \in J$ are the benefit (maximization) objectives

$$
f_{i}(\boldsymbol{x}), i \in I \text { are the cost (minimization) objectives }
$$

and $\quad I \cup J=\{1,2, \ldots, K\}$

To be able to use the distance family relationship (2) for solving problem (3), $\operatorname{PIS}\left(=\boldsymbol{f}^{*}\right)$ and $\operatorname{NIS}\left(=\boldsymbol{f}^{-}\right)$should be determined as follows:

$\begin{aligned} \boldsymbol{f}^{*} & =\left\{\max _{\boldsymbol{x} \in X} f_{j}(\boldsymbol{x}), \forall j \text { AND/OR } \min _{\boldsymbol{x} \in X} f_{i}(\boldsymbol{x}), \forall i\right\}, \text { and } \\ \boldsymbol{f}^{-} & =\left\{\min _{\boldsymbol{x} \in X} f_{j}(\boldsymbol{x}), \forall j A N D / O R \max _{\boldsymbol{x} \in X} f_{i}(\boldsymbol{x}), \forall i\right\}\end{aligned}$

where, $j \in J$ and $i \in I$,

$$
\begin{aligned}
& \boldsymbol{f}^{*}=\left\{f_{1}^{*}, f_{2}^{*}, \ldots, f_{k}^{*}\right\} \text { and } \\
& \boldsymbol{f}^{-}=\left\{f_{1}^{-}, f_{2}^{-}, \ldots, f_{k}^{-}\right\}
\end{aligned}
$$

$f^{*}$ and $f^{-}$are the point solutions in the K-dimensional objective function space. Then, according to PIS and NIS the following distance functions $\mathrm{D}$ from them are obtained as follows:

$d_{p}^{P I S}=\left\{\begin{array}{l}\left.\sum_{j \in J} w_{j}^{p}\left[\left(f_{j}^{*}-f_{j}(\boldsymbol{x})\right) /\left(f_{j}^{*}-f^{-}\right)\right]^{p}+\right]^{\frac{1}{p}} \\ \sum_{i \in I} w_{i}^{p}\left[\left(f_{i}(\boldsymbol{x})-f_{i}^{*}\right) /\left(f_{i}^{-}-f_{i}^{*}\right)\right]^{p}\end{array}\right\}$
$d_{p}^{N I S}=\left\{\begin{array}{l}\left.\sum_{j \in J} w_{j}^{p}\left[\left(f_{j}(\boldsymbol{x})-f_{j}^{-}\right) /\left(f_{j}^{*}-f^{-}\right)\right]^{p}+\right]^{\frac{1}{p}} \\ \sum_{i \in I} w_{i}^{p}\left[\left(f_{i}^{-}-f_{i}(\boldsymbol{x})\right) /\left(f_{i}^{-}-f_{i}^{*}\right)\right]^{p}\end{array}\right\}$

where, $w_{k}, \forall k$ are the relative weights of the corresponding objectives. 
In order to obtain a compromise solution, problem (3) can be transformed to the following bi-objective problem:

$\min d_{p}^{P I S}(\boldsymbol{x})$

$\max d_{p}^{N I S}(\boldsymbol{x})$

subject to $\boldsymbol{x} \in X=\left\{\boldsymbol{x} \mid g_{s}(\boldsymbol{x})\{\geq,=, \leq\} 0, s=1,2, \ldots, m\right\}, p \geq 1 \ldots$ (6)

Since the two objectives of problem (6) may be in conflict with each other one may not be able to simultaneously obtain their individual optima as a single solution. So, a compromise solution has to be obtained and can be obtained by the Closeness Rating Approach. The closeness rating of an alternative/decision is defined as:

$$
C R(\boldsymbol{x})=d_{p}^{N S}(\boldsymbol{x}) /\left(d_{p}^{N S S}(\boldsymbol{x})+d_{p}^{P I S}(\boldsymbol{x})\right)
$$

The nature of the function $\mathrm{CR}(\mathbf{x})$ is such that when an alternative is closest to the Positive Ideal Solution the value of CR tends to 1 . CR tends to 0 when the alternative is closest to the Negative Ideal Solution. The compromise solution of problem (6) then can be solved by the following auxillary problem:

$\operatorname{Max} C R(\boldsymbol{x})=d_{p}^{N I S}(\boldsymbol{x}) /\left(d_{p}^{N I S}(\boldsymbol{x})+d_{p}^{P I S}(\boldsymbol{x})\right)$

subject to $\boldsymbol{x} \in X=\left\{\boldsymbol{x} \mid g_{s}(\boldsymbol{x})\{\geq,=, \leq\} 0, s=1,2, \ldots, m\right\}, p \geq 1 \ldots$.(7)

\section{B. Analytic Hierarchy Process}

The Analytic Hierarchy Process (AHP) [9] is a MCDM technique which attempt to rank a finite set of predetermined alternatives under a finite number of performance criteria. It tries to elicit the hidden preference of the decision makers by asking them to specify the preferences among the various alternatives on each criteria and among criteria themselves by pairwise comparison scheme. A pair of factors can either be two alternatives under specific criteria or two criteria themselves. The decision maker is requested to specify his or her preference for one factor over the other in a ratio scale in a multiplicative AHP. These preferences are then combined to find the overall ranking of the alternatives.

A ratio scale is selected with $\mathrm{n}$ points $1,2, \ldots, \mathrm{n}$ where each of these points represents the level of importance of one factor over the other in a pair wise comparison scheme. The two terminal points 1 and $\mathrm{n}$ of the scale represent equal importance and maximum importance respectively. Accordingly, if there are two factors, $\mathrm{A}$ and $\mathrm{B}$, and if $\mathrm{A}$ gets $\mathrm{m}$ in the scale when comparing with $\mathrm{B}$, it indicates $\mathrm{A}$ is $\mathrm{m}$ times more important than $\mathrm{B}$ and $\mathrm{B}$ will be $1 / m$ times important than $\mathrm{A}$.

\section{APPLICATIONS}

We have considered two example networks with different requirements. In the first one $\left(\right.$ Network $\left._{1}\right)$, we have assumed that medium utilization is most preferred criteria in order to provide more number of simultaneous accesses to multiple users. In the second one $\left(\right.$ Network $\left._{2}\right)$, we have assumed that longevity is the most preferred criteria to have a better communication quality. The objective here is to show that depending on preference assigned to different criteria, the solution changes to get optimal performance.
Using AHP discussed above, we have evaluated the relative weight factors in each case as shown in tables II, III.

For both the network, we have varied the node density (ND) from 50 nodes to 300 nodes per square kilometer. The average speed (v) of the nodes are taken as 10 meters per second. Following values are taken for the parameters of the problems:

$$
\begin{aligned}
& \mathrm{R}_{\max }=200 \text { meters; } \mathrm{R}_{\min }=100 \text { meters; } \\
& \alpha_{\max }=(2 \pi) ; \alpha_{\min }=\pi / 6 ; \mathrm{N}_{\max }=8, \mathrm{~N}_{\min }=1 . \\
& \mathrm{K}_{0}=1 ; \mathrm{K}_{1}=10 ; \mathrm{K}_{2}=0.1 ; \mathrm{K}_{3}=10 ; \mathrm{K}_{4}=1 ; \mathrm{K}_{5}=5 \\
& \mathrm{ND}=50,100,200,300 .
\end{aligned}
$$

Substituting the above parameters , the optimization problems become:

Maximize $\mathrm{L}$, for $\mathrm{v}=10$ metres/second

Maximize $\mathrm{MU}=2 /\left(\mathrm{R}^{2} \alpha\right)$

Minimize $\mathrm{CA}=10+0.1 \mathrm{R}+10 / \alpha$

Minimize $\mathrm{CO}=1+5 / \alpha$

Subject to

$$
\begin{aligned}
& 100 \leq R \leq 200 \\
& \pi / 6 \leq \alpha \leq 2 \pi \\
& 1 \leq N D \cdot R^{2} \alpha / 2 \leq 8
\end{aligned}
$$

It is assumed that a zone should at least contain a node at the given node density. The maximum number of nodes in a zone is assumed to be 8 . The first stage of the analysis is to determine the relevant weight of objectives by multiplicative AHP for the Network 1 and Network ${ }_{2}$ respectively using 5point ratio scale and the results are shown in Table II and III, where RW stands for Raw Weight and NW stands for Normalized weight. Consistency checks have been done [9].

TABLE II

Relative Weight of ObJectives FOR NETWORK 1

\begin{tabular}{|c|c|c|c|c|c|c|}
\hline Objective & L & MU & CA & CO & RW & NW (w) \\
\hline L & 1 & $1 / 5$ & $1 / 2$ & 2 & 0.67 & 0.13 \\
\hline MA & 5 & 1 & 4 & 5 & 3.16 & 0.60 \\
\hline CA & 2 & $1 / 4$ & 1 & 2 & 1.00 & 0.19 \\
\hline CO & $1 / 2$ & $1 / 5$ & $1 / 2$ & 1 & 0.47 & 0.09 \\
\hline TOTAL & $8(1 / 2)$ & $1(13 / 20)$ & 6 & 10 & 5.30 & 1.00 \\
\hline
\end{tabular}

Consistency Ratio, $\mathrm{CR}=2.89 \%<10 \%$

TABLE III

Relative Weight of Objectives for Network $_{2}$

\begin{tabular}{|c|c|c|c|c|c|c|}
\hline Objective & L & MU & CA & CO & RW & NW (w) \\
\hline L & 1 & 3 & 4 & 2 & 2.21 & 0.46 \\
\hline MA & $1 / 3$ & 1 & 3 & $1 / 3$ & 0.76 & 0.16 \\
\hline CA & $1 / 4$ & $1 / 3$ & 1 & $1 / 3$ & 0.41 & 0.08 \\
\hline CO & $1 / 2$ & 3 & 3 & 1 & 1.46 & 0.30 \\
\hline TOTAL & $2(1 / 12)$ & $7(1 / 3)$ & 11 & $3(2 / 3)$ & 4.84 & 1.00 \\
\hline \multicolumn{7}{|c|}{ Consistency Ratio, CR $=4.93 \%<10 \%$}
\end{tabular}

In order to formulate the distance functions $d_{p}^{P I S}$ and $d_{p}$ NIS from the positive ideal solution (PIS) and negative ideal solution (NIS) respectively, for each node density, objective functions $\mathrm{L}, \mathrm{MU}, \mathrm{CA}, \mathrm{CO}$ are to be separately solved for maximum and minimum under common set of constraints to determine $\boldsymbol{f}^{*}$ and $\boldsymbol{f}$. Solving these for the given node densities, the following Table IV is formed.

Using Tables II, III and IV, we form the distance functions $d_{p}^{P I S}$ and $d_{p}{ }^{N I S}$ for different applications at different node densities. 
Table IV

RANGe OF EACH CRITERION $\left(\left|f^{*}-f^{-}\right|\right)$

\begin{tabular}{|c|c|c|c|c|}
\hline $\begin{array}{c}\text { Node } \\
\text { Density }\end{array}$ & L & MU & CA & CO \\
\hline 50 & 15.96 & $4.2 * 10^{-5}$ & 27.51 & 8.75 \\
\hline 100 & 21.29 & $8.75^{*} 10^{-5}$ & 27.51 & 8.75 \\
\hline 200 & 18.12 & $17.50^{*} 10^{-5}$ & 27.51 & 8.75 \\
\hline 300 & 14.92 & $26.25 * 10^{-5}$ & 27.22 & 8.75 \\
\hline
\end{tabular}

Taking $\mathrm{p}=2$, the Euclidean distance functions $d_{p}{ }^{P I S}$ and $d_{p}{ }^{N I S}$ for different applications and node densities can be formulated. As an example, the $d_{p}^{P I S}, d_{p}{ }^{N I S}$ and TOPSIS for both Network 1 and Network 2 for node density 200 can be represented as follows:

Network $_{1}$ for $\mathrm{ND}=200$

$d_{p}^{P I S}(R, \alpha)=\left[\begin{array}{l}\left\{6.96 \times 10^{-3}[19.76-L]\right\}^{2}+\left\{3405.71\left[20 \times 10^{-5}-2 /\left(R^{2} \alpha\right)\right]\right\}^{2}+ \\ \left\{6.87 \times 10^{-3}[0.1 R+10 / \alpha-11.59]\right\}^{2}+\left\{10.17 \times 10^{-3}[5 / \alpha-0.80]\right\}^{2}\end{array}\right]^{1 / 2}$
$d_{p}^{N S}(R, \alpha)=\left[\begin{array}{l}\left\{6.96 \times 10^{-3}[L-1.64]\right\}^{2}+\left\{3405.71\left[2 /\left(R^{2} \alpha\right)-2.5 \times 10^{-5}\right]\right\}^{2}+ \\ \left\{6.87 \times 10^{-3}[39.10-0.1 R-10 / \alpha]\right\}^{2}+\left\{10.17 \times 10^{-3}[9.55-5 / \alpha]\right\}^{2}\end{array}\right]^{1 / 2}$

Network $_{2}$ for $\mathrm{ND}=200$

$d_{p}^{P I S}(R, \alpha)=\left[\begin{array}{l}\left\{25.28 \times 10^{-3}[19.76-L]\right\}^{2}+\left\{897.14\left[20 \times 10^{-5}-2 /\left(R^{2} \alpha\right)\right]\right\}^{2}+ \\ \left\{3.05 \times 10^{-3}[0.1 R+10 / \alpha-11.59]\right\}^{2}+\left\{34.39 \times 10^{-3}[5 / \alpha-0.80]\right\}^{2}\end{array}\right]^{1 / 2}$

$d_{p}^{N S}(R, \alpha)=\left[\begin{array}{l}\left\{25.28 \times 10^{-3}[L-1.64]\right\}^{2}+\left\{897.14\left[2 /\left(R^{2} \alpha\right)-2.5 \times 10^{-5}\right]\right\}^{2}+ \\ \left\{3.05 \times 10^{-3}[39.10-0.1 R-10 / \alpha]\right\}^{2}+\left\{34.39 \times 10^{-3}[9.55-5 / \alpha]\right\}^{2}\end{array}\right]^{1 / 2}$

TOPSIS Formulation for both the networks:

Maximize $C R(R, \alpha)=\frac{d_{p}^{N I S}(R, \alpha)}{d_{p}^{N I S}(R, \alpha)+d_{p}^{P I S}(R, \alpha)}$

Subject to

$100 \leq R \leq 200$

$\pi / 6 \leq \alpha \leq 2 \pi$

$1 \times 10^{4}: R^{2} a \leq 8 \times 10^{4}$

The results are shown in figure 2 and 3.

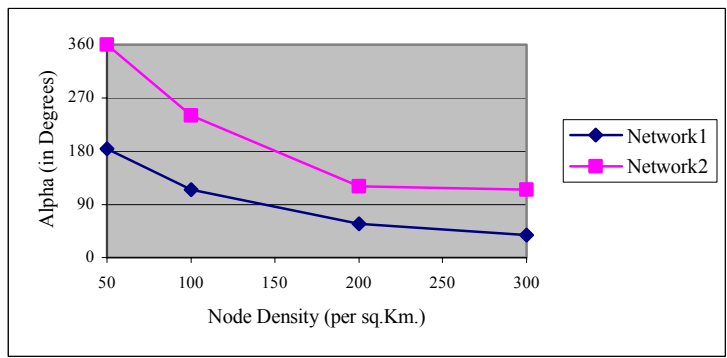

Figure 2: Variation of $\alpha$ with Node Density

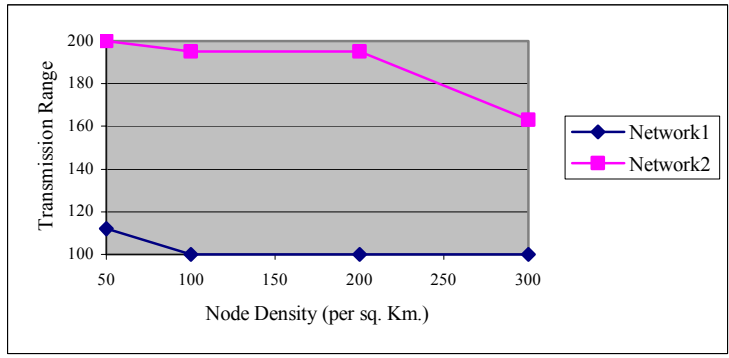

Figure 3: Variation of Transmission Range with Node Density

\section{DISCUSSIONS AND CONCLUSION}

The results show that overall system performance in every context cannot be improved just by using directional antenna. This implies that, a directional antenna capable of forming a narrow beam may not be effective in every scenario to improve the system performance. For example, if improved medium utilisation is the most preffered criteria in an application (network1), then, at high node density (300 per sq. $\mathrm{km}$.), low value of $\alpha\left(38.2^{\circ}\right)$ will result in optimum performance. However, at low node density (50 per sq.km.), low value of $\alpha$ will degrade the system performance and we need to set $\alpha$ at around $183.4^{\circ}$ to get optimum performance (figure 2). In the second example (Network 2 ) where longevity is the most preferred criteria to have a better communication quality, low value of $\alpha$ is unacceptable. We even need an $\alpha$ of $360^{\circ}$ at low node density (50 per sq.km.) at a transmission range 200, indicating that both $\alpha$ and $\mathrm{R}$ need to be at their maximum setting. So, to have a better longevity, both $\mathrm{R}$ and $\alpha$ should be higher (figure 2 and 3 ). In the same example, at higher node density (200-300), $\alpha$ is fixed at $115^{\circ}$ while the transmission range falls from 195 meters at node density 200 to 163 meters at node density 300 (figure 3 ). This is because that $\alpha$ cannot be reduced below $115^{\circ}$ to maintain the high longevity requirement. By lowering $\mathrm{R}$, the optimisation criteria at $\mathrm{ND}=300$ has been fulfilled. It is interesting to note that in most of the cases, $\alpha$ is less than 360 degree indicating that directional antenna is most of the time effective compared to omni-directional antenna. However, the optimum value of $\alpha$ and the corresponding value of $\mathrm{R}$ vary, depending on the application-specific scenario. Depending on preference assigned to different criteria, the solution changes for each application to get optimal performance.

\section{REFERENCES}

[1] E. M. Royer and C-K Toh, "A Review of Current Routing Protocols for Ad hoc Wireless Networks", IEEE Personal Communication, April 1999.

[2] S. Bandyopadhyay, K. Hasuike, S. Horisawa, S. Tawara, "An Adaptive MAC Protocol for Wireless Ad Hoc Community Networ Using Electronically Steerable Passive Array Radiator Antenna", Proc of the GLOBECOM 2001, November 25-29, 2001, San Antonio, Texas, USA.

[3] T.-S. Yum and K.-W. Hung, "Design algorithms for multihop packet radio networks with multiple directional antennas stations," IEEE Transactions on communications, vol. 40, no. 11, pp. 1716--1724, 1992.

[4] Y.-B. Ko, V. Shankarkumar and N. H. Vaidya, "Medium access control protocols using directional antennas in ad hoc networks," Proc. Of the IEEE INFOCOM 2000, March 2000.

[5] A. Nasipuri, S. Ye, J. You and R.E. Hiromoto, "A MAC Protocol for Mobile Ad Hoc Networks Using Directional Antennas", Proc of the IEEE WCNC 2000.

[6] T. Ohira and K.Gyoda, "Electronically Steerable Passive Array Radiator (ESPAR) Antennas for Low-cost Adaptive Beam forming", IEEE Intl. Conference on Phased Array Systems, Dana Point, CA May 2000

[7] Hwang, C.L., and Yoon, K., "Multiple Attribute Decision Making: Methods and Applications", Springer Verlog, 1981

[8] Lai, Y.J., and Hwang, C.L., "Fuzzy Multiple Objective Decision Making : Methods and Applications" Springer Verlog, 1996

[9] Saaty, T.L., "The Analytic Hierarchy Process", McGraw-Hill, 1980 